\title{
Delle variabili complesse sopra una superficie qualunque.
}

\author{
(del prof. Fugenio Bertrami, a Bologna).
}

I.

Rappresentiamo con

$$
d s^{2}=E d u^{2}+2 F d u d v+G d v^{2}
$$

il quadrato dell'clemento lineare della superficic $S$ che dobbiamo considerare.

Non sarà inutile il rammentare fin dal principio cho quando si riguarda una superficie come definita dalla sola espressione dol suo elemento lineare, bisogna prescindere da ogni concetto od imagine che implichi una concreta determinazione della sua forma in relazione ad oggetti esterni, p. es. rispetto ad un sistema d'assi rettangolari. Ogni concetto di questo genere conduce facilmente ad equivoci. Ció solo cho si deve tenere per fermo è che ogni coppia distinta di valori delle variabili $u, v$ individua un punto (o più punti discreti) della superficie, il quale (o ciascuno dei quali) rimane, per sè stesso, essenzialmente distinto da quello (o da ciascuno di quelli) cui corrisponde un'altra coppia di valori, non identica alla prima. La possibilità della coincidenza, in un medesimo luogo dello spazio, di due punti non aventi le stesse coordinate curvilinee, non interviene propriamente che quando si considera, 0 si sottintende, una determinata configurazione della superficie.

La natura delle linee $u=c o s t$., $v=$ cost. rimane sostanzialmente indeterminata; ma, per la precisione degli enunciati, noi non considereremo della superficie $S$ che una porzione $\Omega$, continua e finita, entro la quale si trovino adempiute le seguenti condizioni: $1^{\circ}$ che le funzioni $E, F, G$ sieno in ogni punto di $\Omega$ reali, monodrome, continue c finite; $2^{0}$ she lo funzioni $E, G$, 
$E G-F^{2}\left(=H^{2}\right)$ ricevano in ogni punto di $\Omega$ valori positivi e diversi da zero, talchè anche i radicali $\sqrt{E}, \sqrt{\vec{G}}, \sqrt{E G-F^{2}}(=H)$, che prenderemo sempre positivamente, si conservino reali e monodromi entro l'area considerata.

Di queste condizioni alcune sono necessarie perchè i punti di $\Omega$ sieno tutti reali; le altre impongono certe restrizioni alla natura del doppio sistema di curve coordinate, entro i limiti di $\Omega$. Infatti, avendosi in generale:

$$
\cos \theta=\frac{F}{\sqrt{E G}}, \quad \operatorname{sen} \theta=\frac{H}{\sqrt{E G}},
$$

dove $\theta$ è l'angolo delle due curve intersecantisi nel punto $(u, v)$, l'ipotesi che $\sqrt{E}, \sqrt{G}$ ed $H$ sieno quantità positive e maggiori di zero esclude il caso che l'angolo $\theta$ diventi $=0^{\circ}$ oppure $=180^{\circ}$, cioè che due curve di diverso sistema si tocchino, entro l'area $\Omega$. Conseguentemente la distanza normale di due curve infinitamente vicine del sistema $u=$ cost. (oppure $v=$ cost.) ha un rapporto finito coll' elemento $d v \sqrt{G}$ (oppure $d u \sqrt{E}$ ) che esse intercettano sulla curva dell'altro sistema passante pel punto considerato, e poichè questo elemento è (per le ipotesi) infinitesimo dello stess'ordine di $d v$ (oppure di $d u$ ), ne emerge che ad incrementi infinitesimi del parametro $u$ (oppure $v$ ) corrispondono curve che sono fra loro infinitamente vicine, ma che non hanno alcun punto comune, nell'interno di $\Omega$.

Inoltre, poichè $\sqrt{E}, \sqrt{\dot{G}}$ ed $H$ sono funzioni monodrome in tutti i punti di $\Omega$, lo stesso ha luogo per le espressioni di $\cos \theta$ e $\operatorname{sen} \theta$ : quindi è impossibile che ad una medesima coppia di valori delle $u, v$ corrispondano due valori dell'angolo $\theta(*)$, e conseguentemente che una linea dell'un sistema tagli quelle doll'altro in più di un punto interno ad $\Omega$, oppure che una curva di qualsivoglia sistema si intersechi con sè stessa in un tal punto.

Ammesse queste proprieta, conseguenze delle ipotesi stabilite, ne risulta evidentemente che ciascuna piccola regione della superficie, circostante ad un punto situato nell'interno di $\Omega$, è coperta da un reticolo di curve coordinate il quale, salvo deviazioni minime, è in tutto simile a quello formato sopra un piano da due sistemi di rette parallele. Una tal regione si puó chiamare ordinaria. Cosi, l'area $\Omega$ e tutta formata di regioni ordinarie,

(*) Quand' anche si volessero supporre eguali questi due valori, non cesserebbe di sussistore la stessa impossibilità, perchè variando infinitamente poeo la disposizione delle curve (senza alterare le loro condizioni generali) si renderebbero disuguali i due angoli, senza togricro la monodromia delle funzioni $\operatorname{sen} \theta+\cos \theta$ 
mentre invece il reticolo formato sopra un piano da un sistema di rette divergenti dal centro comune di un sistema di circonferenze concentriche, non presenta dovunque questo carattere, benchè lo riacquisti coll'escludere semplicemente la regione immediatamente circostante al polo.

Per brevità di linguaggio chiameremo curva $u$ (oppure curva $v$ ) relativa ad un punto dato $\left(u_{0}, v_{0}\right)$ della superficie, quella lungo la quale si ha $v=v_{0}$ (oppure $u=u_{0}$ ), mentre $u$ (oppure $v$ ) è variabile lungo la medesima. La direzione positiva della curva $u$ (oppure della curva $v$ ) è quella dello spostamento prodotto da un incremento positivo dato al valore di $u$ (oppure di $v$ ), e quindi quella stessa nella quale cresce il suo arco, il cui incremento è $d s_{u}=d u \sqrt{E}$ (oppure $d s_{v}=d v \sqrt{G}$ ). La direzione positiva di una curva $u$ (oppure $v$ ), nei varii suoi punti interni ad $\Omega$, non puó mai cambiare di senso dall'uno all'altro, perchè $E$ (oppure $G$ ) non puó mai, per ipotesi, diventare $=0$. Ne risulta che, se in un punto qualunque interno ad $\Omega$, si conducono le tangenti alle due curve coordinate, nelle rispettive direzioni positive, e si fa poscia muovere il punto entro $\Omega$ in modo continuo (del resto arbitrario) insieme colle due tangenti positive, mobili intorno ad esso come due aste riunite a corniera, ha luogo la proprietà che queste due tangenti conservano sempre la medesima disposizione relativa, cioè che il senso della rotazione atta a condurre la tangente della curva $u$ su quella della curva $v$ attraverso l'angolo $\theta\left(<180^{\circ}\right)$ interposto e sempre il medesimo. Infatti esso non potrebbe mutare che o con continuità, o per salto: ma non puỏ mutare con continuità perchè l'angolo $\theta$ non puó mai raggiungere nè $0^{\circ}$ nè $180^{\circ}$, come si è già veduto; e neppure puó mutare per salto, perchè la direzione positiva della tangente si mantiene costante lungo ciascuna curva $u \circ v$, come si è pure osscrvato or ora. Conseguentemente se si conduce una normale alla superficie, dalla parte conveniente acciò essa si trovi disposta rispetto alle tangenti delle curve $u v$ come l'asse delle $z$ lo è rispetto a quelli delle $x$ e delle $y$, si vede che questa normale positiva non puo mai mutare di senso, nell'interno di $\Omega$, epperó, assumendo come faccia positiva della superficie quella sulla quale e eretta la normale positiva, un punto il quale sia mobile comunque con continuità, entro i limiti di $\Omega$ non deve mai attraversare la superficie per mantenersi sulla sua faccia positiva. Solamente fa d'uopo notare che se l'area $\Omega$ è composta di più pczzi distinti (circostanza che non è esclusa dalle ipotesi fatte) puo accaderc che la faccia positiva di uno di questi pezzi non sia nel prolungamento di quella d'un altro: ma in questo caso il punto 
Beltrami: Delle variabili complesse sopra una superficie ecc.

mobile non potrebbe passare dall' un pezzo all'altro con continuita, e le conclusioni precedenti varrebbero per ciascun pezzo in particolare.

Queste considerazioni, alle quali converrebbe dare una maggiore estensionc, se non fossero qui subordinate ad uno scopo speciale, sono assai utili per togliere di mezzo le difficoltà che potrebbero altrimenti insorgere in certe circostanze.

Completiamo questi preliminari col richiamare alcune formole ed espressioni notc.

Abbiansi due elementi lineari $d s, \delta s$ uscenti dal punto $(u, v)$, al primo dei quali corrispondano le variazioni $d u$, $d v$, al secondo le $\delta u$, $\delta v$, e sia $\varepsilon$ l'angolo che formano, contato, nel senso positivo, da $d s$ verso $\delta s$. Qualunque sia il valore di $\varepsilon$, si hanno le seguenti formole, facilmente dimostrabili:

$$
\left.\begin{array}{l}
d s \delta s \cos \varepsilon=E d u \delta u+F(d u \delta v+d v \delta u)+G d v \delta v, \\
d s \delta s \text { sen } \varepsilon=H(d u \delta v-d v \delta u) .
\end{array}\right\}
$$

Chiamando $\Delta \omega$ il valore assoluto dell'area del parallelogrammo formato sui due elementi $d s, \delta s$, ed osservando che $\Delta \omega= \pm d s \delta$ sen $\varepsilon$ secondo che $\varepsilon$ è minore o maggiore di $180^{\circ}$, si ha, dalla seconda delle precedenti formole,

$$
\Delta \omega= \pm H(d u \delta v-d v \delta u)
$$

formola nella quale si deve scegliere il segno superiore o l'inferiore secondo che la rotazione da $d s$ verso $\delta s$, attraverso l'area del parallelogrammo, avviene nel senso positivo 0 in senso inverso.

Finalmente rammentiamo le espressioni generali di certe quantita cho abbiamo chiamate altrove $\left(^{*}\right)$ parametri differenziali di $1^{\circ}$ di $2^{\circ}$ ordine. So $\phi$ o $\psi$ sono due funzioni di $u$ e di $v$, queste quantità sono lo seguenti:

$$
\left.\begin{array}{l}
\Delta_{1} \phi=\frac{E\left(\frac{\partial \varphi}{\partial v}\right)^{2}-2 F \frac{\partial \varphi}{\partial v} \frac{\partial \varphi}{\partial u}+G\left(\frac{\partial \varphi}{\partial u}\right)^{2}}{H^{2}}, \\
\Delta_{1} \phi \psi=\frac{E \frac{\partial \varphi}{\partial v} \frac{\partial \psi}{\partial v}-F\left(\frac{\partial \varphi}{\partial v} \frac{\partial \psi}{\partial u}+\frac{\partial \psi}{\partial u} \frac{\partial \psi}{\partial u}\right)+G \frac{\partial \varphi}{\partial u} \frac{\partial \psi}{\partial u}}{H^{2}}, \\
\Delta_{2} \phi=\frac{1}{H}\left\{\frac{\partial}{\partial u}\left(\frac{G \frac{\partial \varphi}{\partial u}-F^{\prime} \frac{\partial \varphi}{\partial v}}{H}\right)+\frac{\partial}{\partial v}\left(\frac{E \frac{\partial \varphi}{\partial v}-F \frac{\partial \varphi}{\partial u}}{H}\right)\right\}
\end{array}\right\}
$$

(*) Rieerche di analisi applicatu alla geometria, nel Giornale di Matematiche di Napoli. Qui abbiamo leggrermente nutate le segnature e le definizioni. 
Beltrami: Delle variabili complesse sopra una superficie eec. 333

$\Delta_{1} \phi$ e $\Delta_{2} \phi$ sono rispettivamente i parametri differenziali di $1^{\circ}$ e $d i 2^{\circ}$ ordine della funzione $\phi ; \Delta_{1} \phi \psi$ è il parametro intermedio delle due funzioni $\phi$ e $\psi$, che si riduce ad un parametro di $1^{\circ}$ ordine quando le due funzioni sono eguali. Fra le proprietà di questi parametri, oltre quella comune della loro invariabilità (vedi le citate Ricerche), ricorderemo le seguenti: $1^{\circ}$ Che chiamando $\delta n$ la distanza normale delle duc curve $\phi$ e $\phi+\delta \phi$ nel punto $(u, v)$ si ha

$$
\Delta_{1} \phi=\frac{\delta \varphi^{2}}{\delta n^{2}}
$$

$2^{\circ}$ Che ponendo $=0$ il parametro intermedio $\Delta_{1} \phi \psi$ si ha la condizione di ortogonalità dei due sistemi di curve $\phi=$ cost., $\psi=$ cost. $3^{\circ}$ Che ponendo $=0$ il parametro di $2^{\circ}$ ordine $\Delta_{2} \phi$ si ha la condizione di isometria del sistema di curve $\phi=$ cost, cioè la condizione perchè le varie curve di esso, corrispondenti ad incrementi infinitesimi ed eguali di $\phi$, associate colle curvo ortogonali (opportunamente distribuito), dividano la superficie in quadrati infinitamente piccoli.

II.

Siano

$$
U d u+V d v, \quad U d u+V^{\prime} d v
$$

i duc fattori imaginari conjugati del secondo membro della (1), dove

$$
U=\sqrt{E}, \quad V=\frac{F+i H}{U}, \quad V^{\prime}=\frac{F-i H}{U}
$$

e quindi $V V^{\prime}=G$. Conserviamo alle caratteristiche $d, \delta \mathrm{i}$ significati in cui furono adoporati nel precedente articolo, e formiamo il rapporto

$$
\frac{U \delta u+V \delta v}{U d u+V d v}
$$

al quale si può dare la forma $\rho e^{i \lambda}$, talchè

$$
\frac{E \delta u+F \delta v+i H \delta v}{E d u+F d v+i H d v}=\rho e^{i \lambda},
$$


Eguagliando fra loro le parti reali ed imaginarie di questa equazione, e ponendo mente alle (2), si trova

$$
\rho \cos \lambda=\frac{\delta s}{d s} \cos \varepsilon, \quad \rho \operatorname{sen} \lambda=\frac{\delta s}{d s} \operatorname{sen} \varepsilon,
$$

donde

$$
\rho=\frac{\delta s}{d s}, \quad \lambda=\varepsilon
$$

e quindi

$$
\frac{U \delta u+V \delta v}{\delta s}=\frac{U d u+V d v}{d s} e^{i \varepsilon}
$$

Se supponiamo che le lunghezze $d s, \delta$ s sieno eguali fra loro, il secondo elemento $\delta s$ pud considerarsi come ottenuto mediante una rotazione $=\varepsilon$ del primo, nel senso positivo. La formola precedente ci insegna pertanto che il binomio differenziale complesso $U \delta u+V \delta v$, relativo all'elemento ruotato, si ottiene dall'analogo binomio $U d u+V d v$ relativo all' elemento primitivo, moltiplicando quest' ultimo pel fattore $e^{i \varepsilon}$ dove $\varepsilon$ è la grandezza della rotazione: proprieta analoga a quella che ha luogo nel piano pel binomio finito $x+i y$, considerato come rappresentante un raggio vettore uscente dall'origine delle coordinate. La medesima proprietả ha luogo naturalmente anche se il binomio $U d u+V d v$ si moltiplica per una funzione qualunque delle $u, v$, e quindi in particolare essa sussiste per il binomio

$$
x(U d u+V d v)=d p+i d q=d w
$$

che si ottiene moltiplicando il binomio prinitivo per uno qualunque, $x$, dei fattori che lo rendono differenziale esatto (fattore generalmente imaginario).

É bene notare che, stante la forma non simmetrica delle quantità $U, V$, allo precedenti relazioni si potrebbe dare un altro aspetto: così la (5) puo scriversi nei due modi seguenti:

$$
\frac{E \delta u+F \delta v+i H \delta v}{E d u+F d v+i H d v}=\frac{F \delta u+G \delta v-i H \delta u}{F d u+G d v-i H d u}=\frac{\delta s}{d s} e^{i \varepsilon},
$$

che è utile di tener presenti, per cvitare delle trasformazioni.

Dalle precedenti osservazioni o facilo rilevare che, quando si voglia applicare vantaggiosamente la teoria delle variabili complesse e delle loro funzioni allo studio delle superficie, non $\delta$ in generale $u+i v$ la variabile complessa che conviene scegliere, ma bensi quella che si ottiene dall'integrazione del binomio 
Beltrami : Delle variabili complesse sopra una superficie ecc. $\mathbf{3 3 5}$

$U d u+V d v$ previamente moltiplicato per un suo fattoro integrante $\boldsymbol{x}$. Ora, benchè la determinazione di una tale variabile complessa dipenda da una integrazione, eseguibile solamente in certi casi particolari, le funzioni di essa, considerate per rapporto alle primitive variabili $u$ e $v$, posseggono dei caratteri speciali, sufficienti a definirle, ed assegnabili in generale a priori.

Sia infatti $f(u, v)$ una funzione della variabile complessa $w$ concepita nel sonso testè dichiarato e riferita alla superficie $S$. Consideriamo la derivata di questa funzione rispetto a quella variabile, derivata che si puo rappresentare così:

$$
\frac{d f}{d u}=\frac{\frac{\partial f}{\partial u} d u+\frac{\partial f}{\partial v} d v}{x(U d u+V d v)},
$$

ossia :

$$
\frac{d f}{d w}=\frac{\frac{\partial f}{\partial u}+\frac{\partial f}{\partial v} \frac{d v}{d u}}{x U+x V \frac{d v}{d u}} .
$$

Se assumiamo, con Rremans, come carattere distintivo delle nuove funzioni, quello di avere una derivata unica, indipendente dalla direzione $\frac{d v}{d t}$, dovremo porre:

$$
\frac{\partial f}{\partial u}: \frac{\partial f}{\partial v}=x U: x V
$$

ossia :

$$
U \frac{\partial f}{\partial v}-V \frac{\partial f}{\partial u}=0
$$

Ia proprietà carattoristica delle funzioni $f$ di $w$ ̀̀ tutta contenuta in questa equazione, la quale puó scriversi nei due modi seguenti:

$$
E \frac{\partial f}{\partial v}-F \frac{\partial f}{\partial u}=i H \frac{\partial f}{\partial t}, \quad G \frac{\partial f}{\partial u}-F \frac{\partial f}{\partial v}=-i I \frac{\partial f}{\partial v} .
$$

Elevando al quadrato ambedue i membri di una di queste equazioni, si trova:

$$
E\left(\frac{\partial f}{\partial v}\right)^{2}-2 F \frac{\partial f}{\partial v} \frac{\partial f}{\partial u}+G\left(\frac{\partial f}{\partial u}\right)^{2}=0
$$


ossia, per le (3),

$$
\Delta_{1} f=0 \text {, }
$$

equazione che sussiste anche per quella funzione che si deduce da $f$ mutando $i$ in $-i$. Dunque: le funzioni $\mathrm{f} d i \mathrm{w}$, espresse per $1 \mathrm{e} \mathrm{v}$, hanno il parametro differenziale di $1^{\circ}$ ordine eguale a zero, e sono le sole che godano di questa proprietà.

Supponiamo decomposta la funzione $f$ nelle sue due parti, reale ed imaginaria, e sia $f=\phi+i \psi$. Sostituendo questo valore nella (8) si trova facilmente:

$$
\Delta_{1} \phi-\Delta_{1} \psi+i \Delta_{1} \phi \psi=0
$$

donde, poichè $\phi$ e $\psi$ sono funzioni reali, si trae:

$$
\Delta_{1} \phi \psi=0, \quad \Delta_{1} \phi=\Delta_{1} \psi
$$

La prima di queste due equazioni esprime (art. I) che: le due famiglie di curve $\phi=$ cost. $\psi=$ cost. sono fra loro ortogonali. La seconda può scriversi (ibid.)

$$
\left(\frac{\delta \varphi}{\delta \downarrow}\right)^{2}=\left(\frac{\delta n_{\varphi}}{\delta n_{\psi}}\right)^{2}
$$

$\delta n_{\mathscr{q}}, \delta n_{\psi}$ essendo le distanze normali, nello stesso punto $(u, v)$, di due curve contigue del sistema $\phi=$ cost., e di due del sistema $\psi=$ cost. Se ne conclude che: prendendo gli incrementi $\delta \phi, \delta \psi$ eguali fra loro, le curve $\phi=$ cost., $\psi=$ cost. dividono la superficie in quadrati infinitamente piccoli, ciò sono curve isometriche.

Sostituendo nelle ( 7 ) il valore $f=\phi+i \psi$ si trovano lc seguenti quattro equazioni reali :

$$
\left.\begin{array}{ll}
\frac{E \frac{\partial \varphi}{\partial v}-F \frac{\partial \varphi}{\partial u}}{H}=-\frac{\partial \psi}{\partial u}, & \frac{G \frac{\partial \varphi}{\partial u}-F \frac{\partial \varphi}{\partial v}}{H}=\frac{\partial \psi}{\partial v}, \\
\frac{E \frac{\partial \psi}{\partial v}-F \frac{\partial \psi}{\partial u}}{H}=\frac{\partial \varphi}{\partial u}, & \frac{G \frac{\partial \psi}{\partial u}-F \frac{\partial \psi}{\partial v}}{H}=-\frac{\partial \varphi}{\partial v},
\end{array}\right\}
$$

delle quali due qualunque sono una conseguenza dolle rimanenti. Queste formole contengono le relazioni necessarie e sufficienti a caratterizzare le duc parti, reale ed imaginaria, di una funzione $\mathrm{f}$, della specie qui considerata. 
Beltrami: Delle variabili complesse sopra una superficie ecc. $\mathbf{3 3 7}$

Eliminando la funzione $\psi$ si trova:

$$
\frac{\partial}{\partial u}\left(\frac{G \frac{\partial \varphi}{\partial u}-F \frac{\partial \varphi}{\partial v}}{H}\right)+\frac{\partial}{\partial v}\left(\frac{E \frac{\partial \varphi}{\partial v}-F \frac{\partial \varphi}{\partial u}}{H}\right)=0 ;
$$

ed un' equazione della medesima forma ottiensi eliminando $\phi$. Questi due risultati possono scriversi [art. I, eq. (3)]:

$$
\Delta_{2} \phi=0, \quad \Delta_{2} \psi=0 \text {, }
$$

e conseguentemente si ha del pari:

$$
\Delta_{2} f=0 \text {, }
$$

come direttamente si deduce anche dalle (7). Dunque: le funzioni f hanno la proprieta di avere ambedue $i$ parametri differenziali eguali a zero; $e$ le loro due componenti reali $\phi e \psi$ hanno il parametro di $20^{\circ}$ ordine eguale a zero.

Una funzione reale $\phi$, soddisfacente all' equazione $\Delta_{2} \phi=0$, puó sempre considerarsi come la parte reale di una funzione $\mathrm{f}$. Infatti, qualunque sia la funzione reale $\phi$, l'equazione $\phi=$ cost. rappresenterà un sistema di curve, che saranno tagliate ortogonalmente dalle curve di un certo altro sistena $\Psi=$ cost., talchè si avrà:

ossia :

$$
\Delta_{1} \phi \Psi=0
$$

$$
\frac{\partial \Psi}{\partial v}\left(E \frac{\partial \varphi}{\partial v}-F \frac{\partial \varphi}{\partial u}\right)+\frac{\partial \Psi}{\partial u}\left(G \frac{\partial \varphi}{\partial u}-F \frac{\partial \varphi}{\partial v}\right)=0
$$

Esisterà dunque un fattore $K$, funzione in generale di $u, v$, che renderà :

$$
E \frac{\partial \varphi}{\partial v}-F \frac{\partial \varphi}{\partial u}=-K \frac{\partial \Psi}{\partial u}, \quad G \frac{\partial P}{\partial u}-F \frac{\partial \varphi}{\partial v}=K \frac{\partial \Psi}{\partial v}
$$

Sostituendo questi valori nella $\Delta_{2} \phi=0$, si ottiene:

$$
\frac{\partial \frac{K}{H}}{\partial u} \frac{\partial \Psi}{\partial v}-\frac{\partial \frac{K}{H}}{\partial v} \frac{\partial \Psi}{\partial u}=0,
$$

equazione dalla quale si deduce, come ò noto,

$$
\frac{\boldsymbol{K}}{I I}=F(\mathrm{I})
$$

Annali di Matematica, tomo 1. 
dove $F$ rappresenta una funzione arbitraria. Se quindi si pone:

$$
F(\Psi) d \Psi=d \downarrow
$$

ciò che equivale a mutare il parametro delle curve ortogonali alle date, si ha:

$$
F(\Psi) \frac{\partial \Psi}{\partial u}=\frac{\partial \psi}{\partial u}, \quad F(\Psi) \frac{\partial \Psi}{\partial v}=\frac{\partial \psi}{\partial v}
$$

e le equazioni $(\dagger)$ ricadono nelle $(9)$.

III.

Affinche le funzioni complesse $f$ rientrino nelle ordinaric funzioni del binomio $u+i v$, bisogna, come è noto, che sussistano le due relazioni:

$$
\frac{\partial \varphi}{\partial u}=\frac{\partial \psi}{\partial v}, \quad \frac{\partial \varphi}{\partial v}=-\frac{\partial \psi}{\partial u} \text {. }
$$

Eliminando con queste la funzione $\psi$ dalle (9) si trova:

$$
(H-E) \frac{\partial \eta}{\partial v}+F \frac{\partial \varphi}{\partial u}=0, \quad F \frac{\partial \varphi}{\partial u}+(H-G) \frac{\partial p}{\partial u}=0
$$

equazioni che non possono essere soddisfatte simultaneamente da una modesima funzione delle $u, v$ se non ha luogo l'identita:

$$
(H-E)(H-G)-F^{2}=0, \text { ossia }(E-G)^{2}+4 F^{2}=0,
$$

la quale, ritenuta reale la superficie, si decompone nelle due condizioni:

$$
E=G, \quad F=0,
$$

che caratterizzano le coordinate isometriche. Dunque solamente pei varii sistemi di coordinate isometriche hanno luogo, rispetto ad una superficie qualunque, quelle proprietà che si verificano nel piano, riferito a coordinate rettangole $x$ ed $y$, per le funzioni dell'ordinaria variabile complessa $x+i y$.

Tratteniamoci alquanto su questo caso, il quale, benchè già molto conosciuto, porge nondimeno occasione ad alcune nuove considerazioni di qualche interesse. 
Beltrami: Delle variabili complesse sopra una superficie ecc. 339

Ritenuta per l'elemento lineare la forma:

$$
d s^{2}=\frac{d l^{2}+d q^{2}}{k^{2}},
$$

imaginiamo che sulla superficie sia tracciata una linea chiusa, rarpresentata dalle equazioni:

$$
p=p_{0}(u), \quad q=q_{0}(u),
$$

dove $u$ è un parametro che ne individua i successivi punti. Poichè la linea si suppone chiusa, è chiaro che la scclta di questo parametro si poträ sempre fare in modo che le due funzioni $p_{0}(u), q_{0}(u)$ sieno periodiche e che il loro periodo sia $2 \pi$, talchè per mezzo del teorema di Fourrer si potranno esprimere nel modo seguente:

$$
p_{0}(u)=\frac{1}{2 \pi} \sum_{-\infty}^{+\infty} \int_{0}^{2 \pi} p_{0}(\alpha) e^{i m(u-\alpha)} d \alpha, \quad q_{0}(u)=\frac{1}{2 \pi} \sum_{-\infty}^{+\infty} \int_{0}^{2 \pi} q_{0}(\alpha) e^{i n(u-\alpha)} d \alpha .
$$

Ciò premesso, supponiamo diviso l'intero periodo $2 \pi$ della variabile $u$ in un gran numero di parti eguali che denoteremo con $\Delta u$, comispondenti ad altrettanti elementi in cui si troverà divisa la curva e che riguarderemo come rettilinei. Poscia facciamo ruotare ciascun elemento di un angolo $\lambda$ (costante) contato verso l'interno della curva e indichiamo con $\nabla p, \nabla q$ le variazioni di $p, q$ corrispondenti al termine dell' elemento così spostato, conservando la caratteristica $\Delta$ per le variazioni lungo la curva primitiva. Per la proprietà stabilita al principio dell' articolo precedente avremo:

$$
\nabla(p+i q)=e^{i \lambda} \cdot \Delta\left(p_{0}+i q_{0}\right) .
$$

Facendo quest'operazione per tutti gli elementi, avremo, nei termini degli elementi stessi dopo la rotaziove, i punti di una linea contigua alla primitiva, c indicatene con $p_{1}, q_{1}$ le coordinate, potremo scrivere:

$$
p_{1}+i q_{1}=p_{0}+i q_{0}+e^{i \lambda} \Delta\left(p_{0}+i q_{0}\right)
$$

ovvero, usando una notissima segnatura simbolica,

$$
p_{1}+i q_{1}=\left(1+e^{i \lambda} \Delta\right)\left(p_{0}+i q_{0}\right) \text {. }
$$

Operando su questa seconda curva come si è fatto sulla prima, si trova una terza curva, ed indicandone con $p_{2}, q_{2}$ le coordinate, si ha, pure simbolicamente,

$$
p_{2}+i q_{2}=\left(1+e^{i \lambda} \Delta\right)^{2}\left(p_{0}+i q_{0}\right)
$$


340 Beltrami: Delle variabili complesse sopra una superficie ecc.

poscia:

$$
p_{3}+i q_{3}=\left(1+e^{i \lambda} \Delta\right)^{3}\left(p_{0}+i q_{0}\right)
$$

e dopo $n$ simili operazioni :

$$
p_{n}+i q_{n}=\left(1+e^{i \lambda} \Delta\right)^{n}\left(p_{0}+i q_{0}\right)
$$

Ora, se per brevità si pone:

$$
p_{0}(u)+i q_{0}(u)=F(u)
$$

dalle due formole (11) si deduce:

$$
p_{0}+i q_{0}=\frac{1}{2 \pi} \sum_{-\infty}^{+\infty} \int_{0}^{z \pi} F(\alpha) e^{i n(u-\alpha)} d \alpha
$$

e da questa, continuando ad usare la precedente seguatura simbolica,

$$
\left(1+e^{i \lambda} \Delta\right)^{n}\left(p_{0}+i q_{0}\right)=\frac{1}{2 \pi} \sum_{-\infty}^{+\infty}\left(1+m i e^{i \lambda} \Delta u\right)^{n} \int_{0}^{2 \pi} F(\alpha) e^{i m(u-\alpha)} d \alpha .
$$

Il luogo dei punti $\left(p_{n}, q_{n}\right)$ corrispondenti ai successivi valori di $n$ e ad uno stesso valore di $u$, è una curva di cui chiameremo $v$ il parametro, ed è chiaro che potremo porre $v=n . \Delta u$, talchè la curva primitiva sarà rappresentata da $v=0$. Per tal guisa, chiamando $p, q$ le coordinate del punto $(u, v)$ e raffrontando le due equazioni (12) (13), potremo scrivere:

$$
p+i q=\frac{1}{2 \pi} \sum_{-\infty}^{+\infty}\left\{\int_{0}^{2 \pi} F^{\prime}(\alpha) e^{i n(u-\alpha)} d a\right\} \cdot \lim \left(1+m i e^{i \lambda} \Delta u\right)^{\frac{v}{\Delta u}} \text { per } \Delta u=0 .
$$

Ma è noto che:

$$
\lim \left(1-\mathfrak{-} m i e^{i \lambda} \Delta u\right)_{\Delta u=0}^{\frac{v}{\Delta u}}=e^{m i e^{i \lambda}}
$$

quindi :

$$
p+i q=\frac{1}{2 \pi} \sum_{-\infty}^{+\infty} \int_{0}^{2 \pi} F(\alpha) e^{i n\left(u_{\uparrow} v e i \lambda \alpha\right)} d \alpha
$$

ovvero, pel teorema di Fourier,

$$
p+i q=F\left(u+v e^{i \lambda}\right)
$$


Beltrami: Delle variabili complesse sopra una superficie ecc. 341

cioè :

$$
p+i q=p_{0}\left(u+v e^{i \lambda}\right)+i q_{0}\left(u+v e^{i \lambda}\right)
$$

Quest'equazione complessa, decomposta in due reali, determina completamente i due sistemi di curve $u=$ cost., $v=$ cost., in coordinate $p$ e $q$, sistemi la cui natura dipende da quella della curva primitiva e del suo parametro $u$ : questa stessa curva appartiene al sistema $v=c o s t$. e corrisponde al valore $v=0$.

Dalla costruzione infinitesimale che abbiamo effettuata emerge chiaramente che i due sistemi $u=$ cost., $v=$ cost. si tagliano dovunque sotto l'angolo costante $\lambda$. Ciò è confermato dall'osservare che l'equazione (14) dà:

$$
d p^{2}+d q^{2}=\left(\bmod F^{\prime}\right)^{2}\left(d u^{2}+2 d u d v \cos \lambda+d v^{2}\right),
$$

e quindi :

$$
d s^{2}=\left(\frac{\bmod F^{\prime}}{k}\right)^{2}\left(d u^{2}+2 d u d v \cos \lambda+d v^{2}\right)
$$

espressione che, paragonata alla (1), mostra essere appunto $\lambda$ l'angolo delle curve $u=$ cost., $v=$ cost. Anzi se si pon mente alla immediata deduzione di questo risultato dalla sola equazione (14), si vede che ha luogo il seguente teorema: Se p e q sono coordinate isometriche di una superficie, eguagliando il binomio $\mathrm{p}+\mathrm{iq}$ ad una qualunque funzione $d i \mathrm{u}+\mathrm{ve}^{\mathrm{i} \lambda}$ (dove $\mathrm{u}, \mathrm{v}$ sono parametri variabili e $\lambda$ una costante reale), si ottengono due sistemi $d i$ curve $\mathrm{u}=$ cost., $\mathrm{v}=$ cost. che si tagliano dovunque sotto l' angolo $\lambda$. Da questo teorema, nel caso particolare in cui $\lambda=\frac{\pi}{2}$, si ottiene una notissima proposizione di Gsuss. La restrizione introdotta nella precedente analisi, col supporre che la primitiva curva $v=0$ fosse chiusa, non è essenziale al teorema precedente, come appare dall' ultima sua dimostrazione.

Il qual teorema dev'essere completato coll'osservazione che le curve del sistema $\mathrm{v}=$ cost. sono, per una stessa funzione $\mathrm{F}$ di $\mathrm{u}+\mathrm{v} \mathrm{e}^{\mathrm{i} \lambda}$, indipendenti dall' angolo $\lambda$, cosicchè da una medesima funzione si deduce un sistema di curve, accompagnato dai sistemi delle sue trajettorie sotto tutti gli angoli possibili. Ci dispensiamo dal dimostrare questa proprietà, la quale risulta con evidenza dalla costruzione infinitesimale effettuata. Cosi non è necessario dimostrare che le curve $v=$ cost. sono isometriche, e che lo sono parimenti tutti i sistemi $u=$ cost. corrispondenti ai diversi valori di $\lambda$. Vi è pero un ravvicinamento che merita di essere fatto, ed è il seguente:

Dietro quanto precede è chiaro che ogni volta che le coordinate $(p \in q)$ 
di una linea tracciata sulla superficie sono date in funzione di un parametro $u$, viene ad essere determinato un sistema di linee isoterme, del quale essa fa parte, ed il quale alla sua volta determina infiniti altri sistemi, formati dalle trajettorie di esso sotto tutti gli angoli costanti. Ne risulta che per ogni punto della curva primitiva passano infinite curve, le quali fanno colla curva stessa tutti gli angoli possibili e che si possono chiamare per un momento le isoterme di quel punto. Ciò posto, se, sulla curva primitiva, si danno ad $u n$ incrementi consecutivi $=\Delta u$, è ovvio che le coordinate (isometriche) $p_{u}, q_{n}$ del punto finale, sono legate alle coordinate $p_{0}, q_{0}$ del punto iniziale dalla formola simbolica:

$$
p_{1}+i q_{n}=(1+\Delta)^{n}\left(p_{0}+i q_{0}\right)
$$

conseguenza della notissima:

$$
p_{n}=(1+\Delta)^{n} p_{0},
$$

riportata in tutti i Trattati. Ora noi invece abbiamo trovato che un analogo spostamento sulla curva isoterma che parte dal punto $\left(p_{0}, q_{0}\right)$ sotto l'angolo $\lambda$ dà luogo alla formola (12), la quale, nel caso particolare dell' angolo retto, diventa:

$$
p_{n}+i q_{n}=(1+i \Delta)^{n}\left(p_{0}+i q_{0}\right) \text {. }
$$

I secondi membri delle equazioni simboliche (15) (16) (12) differiscono pei fattori rispettivi :

$$
1+\Delta, \quad 1+i \Delta, \quad 1+e^{i \lambda} \Delta,
$$

e la forma dei medesimi conduce jaturalmente all'osservazione che, nello stesso modo che il simbolo $\Delta$ nel primo di essi accenna notoriamente ad una differenziazione nel senso della curva primitiva, cioè ad una differenziazione diretta o reale, cosi il simbolo $i \Delta$ del secondo accenna ad una differenziazione ortogonale od imaginaria, ed il simbolo $e^{i \dot{\lambda}} \Delta$ del terzo ad una differenziazione obliqua o complessa. E, mentre la differenziazione diretta (A) che è I'ordinaria, corrisponde allo spostamento del punto sulla curva primitiva, cosi la seconda $(i \Delta)$ corrisponde allo spostamento lungo l' iso. terma ortogonale e la terza $\left(e^{i \lambda} \Delta\right)$ allo spostamento lungo l'isoterma inclinata dell' angolo indicato dal simbolo di differenziazione complessa. Per tal guisa la differenziazione ordinaria si presenterebbe qui come caso particolare di una operazione più generale, la quale troverebbe nelle precedenti considerazioni geometriche una definizione ed un'imagine altrettanto semplici e chiare quanto quelle della prima. 
Beltrami: Delle variabili complesse sopra una superficie ece. 343

IV.

Le funzioni $p_{0}(u), q_{0}(u)$ usate nelle considerazioni dell' articolo precedente possono assumere moltissime forme diverse, senza che la curva da esse individuata cambi sostanzialmente di natura. A ciascuna delle forme conciliabili con questa condizione corrisponde uno speciale sistema isometrico, di cui essa ia parte, precedentemente designato con $v=$ cost. Si può determinare la forma di quelle funzioni introducendo opportune condizioni. Ne daremo un esempio, supponendo che, insieme alla curva anzidetta, sia data la curva isometrica che deve succederle a distanza infinitesima nel sistema suindicato: ovvero, in altri termini, supponendo che si tratti di determinare un sistema isometrico, del quale facciano parte due curve infinitamente vicine, date ad arbitrio.

Le due curre contigue siano definite dalle equazioni:

$$
\left\{\begin{array} { l } 
{ p = p _ { 0 } ( u ) , } \\
{ q = q _ { 0 } ( u ) , }
\end{array} \quad \left\{\begin{array}{l}
p_{1}=p_{0}(u)+\gamma \mathrm{P}(u), \\
q_{1}=q_{0}(u)+\gamma Q(u),
\end{array}\right.\right.
$$

dove $\gamma$ è una costante infinitamente piccola. Gli incrementi delle coordinate $p, q$ corrispondenti all'estremità dell'elemento $d s$ della prima curva sono $d u \cdot p_{0}^{\prime}(u), d u \cdot q_{0}^{\prime}(u)$; all'incontro gli incrementi corrispondenti all' estremità dell' elemento $\delta$ s compreso fra il punto $(u)$ della prima curva e il punto $(u)$ della seconda sono $p_{1}-p_{0}, q_{1}-q_{0}$ ossia $\gamma \mathrm{P}(u), \gamma Q(u)$. Quindi si ha:

$$
d s=\frac{d u \sqrt{p_{0}^{12}+q_{0}^{12}}}{k}, \quad \delta s=\frac{\gamma \sqrt{\mathrm{p}^{2}}+\overline{Q^{2}}}{k},
$$

ed il seno dell' angolo $\lambda$ compreso dai due elementi $d s, \delta s[$ eq. (2)] ì dato da:

$$
\operatorname{sen} \lambda=\frac{\left(p_{0}{ }^{\prime} \mathrm{Q}-q_{0}{ }^{\mathrm{P}} \mathrm{P}\right) \gamma d u}{k^{2} d s \delta s}
$$

se dunque si chiama $\delta n$ la distanza normale delle due curve nel punto $(u)$, si ha:

$$
\delta n=\delta \operatorname{sen} \lambda=\frac{\left(p_{0}{ }^{\prime} 0-q_{0}{ }^{\prime} \mathrm{p}\right) \gamma}{k \sqrt{p_{0}{ }^{\prime}+q_{0}{ }^{\prime}}} \text {. }
$$

Ora, se si vuole che la nuova variabile indipendente, che diremo $w$, determini, coi suoi incrementi eguali. i lati dei quadratelli compresi fra le due curve e gli clementi normali ad esse, bisogna manifestamente che per essa 
344 Beltrami : Delle variabili complesse sopra una superficie ecc.

risulti $d s=\delta n$ cioè :

$$
\frac{d u \sqrt{p_{0}^{\prime 2}+}+\overline{q_{0}^{\prime 2}}}{k}=\frac{\left(p_{0}^{\prime} Q-q_{0}^{\prime} P\right) \gamma}{k \sqrt{p_{0}^{\prime 2}+q_{0}^{18}}}
$$

e, poichè l'infinitesimo $\gamma$ è costante, come dev' esserlo $d w$, si può porre:

$$
\gamma=C d w
$$

$G$ essendo una costante finita. Sostituendo questo valore nella precedente equazione ed integrando si trova:

$$
C w=\int \frac{p_{0}{ }^{2}+q_{0}{ }^{2}}{p_{0}{ }^{\prime} Q-q_{0}{ }^{\prime} \mathrm{p}} d u
$$

quadratura che serve alla determinazione della variabile $w$, la quale non \& altro che il parametro isometrico del sistema ortogonale a quello definito dalle due curve prossime date. La costante $C$ rimane essenzialmente arbitraria, ma nei singoli casi si puó determinare dietro considerazioni di speciale opportunità.

Facciamo due esempi semplicissimi, relativi ad un piano.

Le coordinate isometriche $p, q$ sieno le ordinarie coordinate rettangole $x, y$ e la prima curva sia l' ellisse

$$
\frac{x^{2}}{a^{2}}+\frac{y^{2}}{b^{2}}=1
$$

che rappresenteremo colle due equazioni equivalenti

$$
x=a \cos u, \quad y=b \operatorname{sen} u .
$$

La curva infinitamente vicina sia un'ellisse omofocale. Indicando i semiassi di questa con $a-\alpha, b-\beta(\alpha, \beta$ infinitesimi) si dovrà avere

$$
(\alpha-\alpha)^{2}-(b-\beta)^{2}=a^{2}-b^{2},
$$

quindi

$$
a \alpha=b \beta .
$$

Rappresentando con $c \gamma$ il valor comune di questi due prodotti, dove $\gamma$ c un infinitesimo e $c$ una costante finita, si puo dunque porre

$$
a=\frac{c}{a} \gamma, \quad \beta=\frac{c}{b} \gamma,
$$


Beltrami: Delle variabili complesse sopra una superficie ecc. 345

e l'ellisse contigua alla precedente è rappresentabile colle formole

$$
x_{1}=\left(a-\frac{c}{a} \gamma\right) \cos u, \quad y_{1}=\left(b-\frac{c}{b}\right) \operatorname{sen} u \text {. }
$$

Paragonando le equazioni attuali alle (17) si trova

$$
\begin{array}{ll}
p_{0}=a \cos u, & \mathrm{P}=-\frac{c}{a} \cos u \\
q_{0}=a \operatorname{sen} u, & \mathrm{Q}=-\frac{c}{b} \operatorname{sen} u,
\end{array}
$$

e conseguentemente dalla (18) si trae

$$
w=\frac{C c}{a b} u
$$

0 , piu semplicemente $w=u$, se si determina $C$ in modo che le due variabili $u$ e $w$ vadano contemporaneamente da 0 a $2 \pi$. Per tal modo si vede che le formole atte a rappresentare la prima ellisse dietro la condizione prescritta sono le stesse (19), col semplice cambiamento di $u$ in $w$.

Ciò premesso, se si vuole ottenere il doppio sistema ortogonale $u=c o s t$, $v=$ cost . di cui fanno parte le due ellissi omofocali contigue, basterà fare:

$$
x+i y=a \cos (u+i v)+i b \operatorname{sen}(u+i v)
$$

ovvero, ponendo

$$
a=\sqrt{a^{2}-b^{2}} \cdot \cosh v_{0}, \quad b=\sqrt{a^{2}-b^{2}} \cdot \operatorname{senh} v_{0}
$$

e scrivendo poscia, ciò che è evidentemente lecito, $v$ in luogo di $v_{0}-v$,

$$
x+i y=\sqrt{a^{2}-b^{2}} \cdot \cos (u+i v),
$$

formola donde si ricava, come è notissimo, il doppio sistema ortogonale delle coniche omofocali. Se invece si ponesse

$$
x+i y=\sqrt{a^{2}-b^{2}} \cdot \cos \left(u+v e^{i \hat{u}}\right),
$$

si avrebbe il doppio sistema formato dalle stesse ellissi omofocali del sistema precedente e dalle curve (trascendenti) che le tagliano sotto l'angolo costante $\lambda$.

Il secondo esempio sarà quello di due circonferenze infinitamente vicine, l'una interna all'altra. Ponendo i loro centri sull'asse delle $y$, sia:

$$
x^{2}+(y-a)^{2}=c^{2}
$$


Beltrami: Delle variabili complesse sopra una superficie ecc.

l'equazione della circonferenza esterna, alla quale sostituiremo le formole:

$$
x=c \cos u, \quad y=a+c \operatorname{sen} u .
$$

Indicando con $c-\delta c, a-\delta a$ il raggio e l'ordinata del centro della circonferenza interna, e supponendo $\delta a$ positivo, si deve avere $\delta a<\delta c$, quindi si può porre:

$$
\delta c=\gamma c, \quad \delta a=\gamma c \cos \mu, \quad 0<\mu<\frac{\pi}{2},
$$

e l'equazione della seconda circonferenza risulta, per $\gamma$ infinitesimo,

$$
x^{2}+(y-a)^{2}+2 \gamma c(y-a) \cos \mu=c^{2}-2 c^{2} \gamma,
$$

cosicchè l'asse radicale delle due circonferenze è la retta

$$
(y-a) \cos \mu+c=0 \text {. }
$$

E lecito prendere questa retta per asse delle $x$ ed in questa ipotesi, essendo $y=0$, si trova fra $a$ e $c$ la relazione $c=a \cos \mu$, la quale determina quel punto dell'asse delle $y$ nel quale si deve collocare il centro della prima circonferenza. Se ne conclude che $\mu$ è l'angolo fatto coll'asse delle $x$ dalla tangente condotta a questa dall' origine.

Avuto riguardo ai precedenti risultati, si trova che nel caso attuale si ha:

$$
\begin{array}{ll}
p_{0}=c \cos u, & \mathrm{p}=-c \cos u, \\
q_{0}=c \operatorname{sen} u+\frac{c}{\cos \mu .} & Q=-c\left(\operatorname{sen} u+\frac{1}{\cos \mu}\right),
\end{array}
$$

talche la formola (18) diventa:

$$
C w=\int \frac{d u}{1+\cos u \cdot \operatorname{sen} u} .
$$

Eseguendo l'integrazione ed aggiungendo la condizione che $u$ e $w$ vadano contemporaneamente da 0 a $2 \pi$, si trova:

$$
\operatorname{tg}\left(\frac{u}{2}+\frac{\pi}{4}\right)=\operatorname{tg} \frac{\mu}{2} \cdot \operatorname{tg} \frac{w}{2} .
$$

A questa formola si puod dare la forma:

$$
\frac{e^{i n}+i}{e^{2 n}-i}=i \operatorname{tg} \frac{\mu}{2} \cdot \operatorname{tg} \frac{w}{2}
$$


Beltrami: Delle variabili complesse sopra una superficie ece. 347

e siccome le $(20)$ danno $e^{i u}=\frac{x+i y}{c}-\frac{i}{\cos \mu_{0}}$, cosi si ha pure:

$$
\frac{x+i y-i l \operatorname{tg} \frac{\mu}{2}}{x+i y-i l \operatorname{cotg} \frac{\mu}{2}}=i \operatorname{tg} \frac{\mu}{2} \operatorname{tg} \frac{w}{2},
$$

chiamando $l$ la lunghezza della tangente condotta dall'origine alla circonferenza data, cioè ponendo $l=c \operatorname{tg} \mu$.

Per avere il doppio sistema isometrico ortogonale, bisogna ora porre $u+i v$ in luogo di $w$. Si riduce l'equazione risultante a maggior semplicità cambiando l'origine delle $v$ col porre $u=u+i\left(v-v_{0}\right)$, ossia col cambiare $w$ in $w-i v_{0}$ (se $\left.w=u+i v\right)$. Infatti si trova cosi:

$$
\frac{x+i y-i \lg \frac{\mu}{2}}{x+i y-i \operatorname{cotg} \frac{\mu}{2}}=\operatorname{tg} \frac{\mu}{2} \cdot \operatorname{coth} \frac{v_{0}}{2} \cdot \frac{\lg \frac{v}{2}-i \operatorname{tgh} \frac{v_{0}}{2}}{\operatorname{tg} \frac{v}{2}-i \operatorname{cotgh} \frac{v_{0}}{2}},
$$

ossia, determinando $v_{0}$ in $\bmod 0 \operatorname{chc} \operatorname{tgh} \frac{v_{0}}{2}=\operatorname{tg} \frac{\mu_{0}}{2}$,

$$
\frac{x+i y-i l \lg \frac{\mu}{2}}{x+i y-i l \operatorname{cotg} \frac{\mu}{2}}=\frac{\operatorname{tg} \frac{w}{2}-i \lg \frac{\mu}{2}}{\operatorname{tg} \frac{w}{2}-i \operatorname{cotg} \frac{\mu}{2}}
$$

donde si trac manifestamente:

$$
x+i y=l \operatorname{tg} \frac{u+i \omega}{z}
$$

Ecco dunque come si risolve la questione proposta: Data la circonferenza primitiva di raggio $c$, si faccia passare pel contro di essa e della contigua l'asse delle $y$, e si prenda per asse delle $x$ l'asse radicale delle due circonferenze; indi si determini la lunghezza $l$ della tangente condotta dall'origine, e finalmente si ponga $x+i y=l \operatorname{tg} \frac{u+i v}{2}$. In virtù di tale relazione le variabili $u, v$ diventano i parametri di due sistemi isometrici ortogonali: le due circonferenze primitive appartengono al sistema $v=$ cost., e propriamente l'eq. (20) si ottiene ponendo $v=v_{0}$, dove $\operatorname{tgh} \frac{v_{0}}{2}=V \frac{\overline{a-c}}{a+c}=\frac{a-c}{l}$. 
Il doppio sistema ortogonale cosi ottenuto è formato di due famiglie di circonferenze, ed è tanto noto che è inutile sviluppare ulteriormente la soluzione che vi ci ha condotti, bastando accennare le espressioni delle coordinate ortogonali $x, y$ in funzione delle $u, v$, che sono:

$$
x=\frac{l \operatorname{sen} u}{\cos u+\cosh v}, \quad y=\frac{l \operatorname{senh} v}{\cos u+\cosh v}
$$

e quella dell' elemento lineare, che è:

$$
d x^{2}+d y^{2}=\frac{l^{2}\left(d u^{2}+d v^{2}\right)}{(\cos u+\cosh v)^{2}} .
$$

Se invece si ponesse:

$$
x+i y=l \operatorname{tg} \frac{u+v e^{i \lambda}}{2}
$$

si otterrebbero due sistemi di curve, l'uno dei quali, cioè $v=c o s t$. , sarebbe formato di tutte le circonferenze d'eguale equazione nell'esempio precedente, e l'altro dalle curve che tagliano queste circonferenze sotto l'angolo costante $\lambda$.

V.

Passiamo ora ad un altro genere di considerazioni, che ci servirànno poscia a stabilire lo proprietà del fattore integrante $x$.

Nell'interno dell' area $\Omega$ immaginiamo tracciata una linea chiusa, costituente il contorno completo di un pezzo di superficie appartenente alla regione ordinaria considerata; sia $\Omega^{\prime}$ l'area di questo pezzo.

Se il contorno di $\Omega^{\prime}$ non è per sè stesso tale da essere intersecato in due soli punti da ciascuna delle curve $u=$ cost., $v=$ cost. che lo incontrano, è chiaro che si potrà sempre suddividere l'area $\Omega^{\prime}$ abbracciata da esso, mediante linee $\Lambda^{\prime}$ opportunamente tracciate, in modo che tale condizione sia soddisfatta.

Ciò posto consideriamo l'area $\omega$ di uno dei pezzi risultanti da questa suddivisione, e chiamiamo da il suo elemento, dato da $d \omega=H d u d v$, formola nella quale gli incrementi $d u, d v$ devono essere supposti positivi. Sieno $\phi, \psi$ due funzioni di $u$ e $v$, monodrome, finite e continue, insieme colle 
loro derivate prime, in tutti i punti di $\Omega^{\prime}$, e pongasi:

$$
\Pi=\int \Delta_{1}(\phi \psi) \cdot d \omega
$$

integrale esteso a tutta l'area $\omega$.

Per trasformare agevolmente questo integrale duplicato si ponga, per brevità,

$$
M_{\psi}=\frac{G \frac{\partial \psi}{\partial u}-F \frac{\partial \psi}{\partial v}}{H}, \quad N_{\psi}=\frac{E \frac{\partial \psi}{\partial v}-F \frac{\partial \psi}{\partial u}}{H},
$$

e si avr’a:

$$
\Pi=\iint\left(M_{\psi} \frac{\partial \varphi}{\partial u}+N_{\psi} \frac{\partial \varphi}{\partial v}\right) d u d v=\iint M_{\psi} \frac{\partial \varphi}{\partial u} d u d v+\iint N_{\psi} \frac{\partial \varphi}{\partial v} d u d v
$$

Consideriamo un valore determinato di $v$, e quindi una linea particolare del sistema $v=$ cost, e siano $u=a$. $u=b$ i valori di $u$ corrispondenti ai due punti ai quali il contorno dell'area $\omega$ è incontrato da questa linea $(b>a)$. Per formare l' integrale $\iint M_{\psi} \frac{\partial \varphi}{\partial u} d u d v$ esteso ai limiti noti, bisognerà integrar dapprima rispetto ad $u$, supposto $v=$ al valore fissato, ed estendere questa integrazione fra $u=a$ ed $u=b$. Per l'ammessa continuità delle derivate della funzione $\phi$ si avrà cosi, integrando per parti,

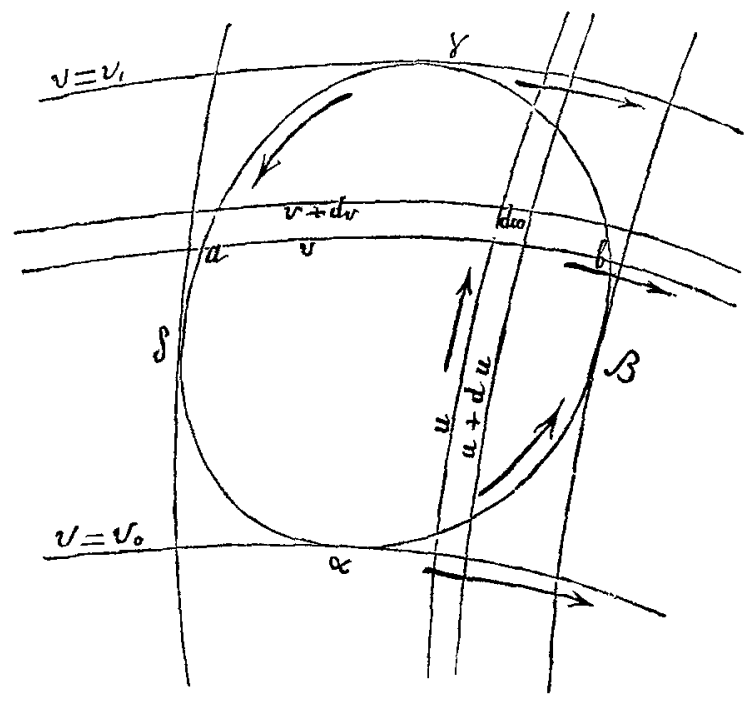

$$
\int_{a}^{b} M_{\psi} \frac{\partial \varphi}{\partial u} d u=\left(M_{\psi} \phi\right)_{u=b}-\left(M_{\psi} \phi\right)_{u=a}-\int_{a}^{b} \frac{\partial M_{\psi}}{\partial u} \phi d u
$$

Le coordinate $u, v$ di un punto qualunque del contorno si possono evidentemente riguardare come funzioni monodrome, finite e continue dell' arco $s$ del suo perimetro, contato da un punto determinato nel senso che si adotta abitualmente come positivo, cioè tale che la normale interna sia disposta rispetto alla tangente positiva come la tangente positiva di una curva $v$ 
lo è rispetto a quella di una curva $u$. Poichè $d v$ è quantità che nell' integrazione si dee riguardare come positiva, si avrà dunque:

$$
\begin{array}{ll}
\text { nel punto } a: & d v=-\left(\frac{d v}{d s}\right) d s, \\
\text { nel punto } b: & d u=+\left(\frac{d u}{d s}\right) d s,
\end{array}
$$

$d s$ essendo sempre quantità positiva: quindi dall'ultima formola ottenuta si potrà ricavare:

$$
\iint M_{\psi} \frac{\partial \psi}{\partial u} d u d v=\int\left(M_{\psi} \psi \frac{d v}{d s}\right)_{a} d s_{a}+\int\left(M_{\psi} \phi \frac{d v}{d s}\right)_{b} d s_{b}-\iint \frac{\partial M_{\psi}}{\partial u} \phi d u d v
$$

Il primo integrale del secondo membro deve manifestamente essere esteso alla serie dei punti analoghi ad $a$, cioè a tutto l'arco $\gamma \delta \alpha$; il secondo, alla serie dei punti analoghi a $b$, cioè a tutto l'arco $\alpha \beta \gamma$ : conseguentemente la loro somma equivale all'integrale unico:

$$
\int M_{\psi} \phi \frac{d v}{d s} d s
$$

esteso lungo tutto il perimetro, percorso nel senso positivo. Dunque si ha la formola :

$$
\iint M_{\psi} \frac{\partial \psi}{\partial u} d u d v=\int M_{\psi} \phi\left(\frac{d v}{d s}\right) d s-\iint \frac{\partial M_{\psi}}{\partial u} \bar{\phi} d u d v
$$

nella quale $i$ due integ'rali duplicati sono estesi a tutta l'area $\omega$ e l'intcgrale semplice a tutto il contorno, percorso nel senso positivo.

Procedendo analogamente per l'altra parte dell' integrale $\Pi$ si trova:

$$
\iint N_{\psi} \frac{\partial \varphi}{\partial v} d u d v=-\int N_{\psi} \phi\left(\frac{d u}{d s}\right) d s-\iint \frac{\partial N_{\psi}}{\partial v} \phi d u d v
$$

e quindi, sommando membro a membro,

$$
\Pi=\int\left\{M_{\psi}\left(\frac{d v}{d s}\right)-N_{\psi}\left(\frac{d u}{d s}\right)\right\} \phi d s-\iint\left(\frac{\partial M_{\psi}}{\partial u}+\frac{\partial N_{\psi}}{\partial v}\right) \phi d u d v .
$$

Ma si ha:

$$
\frac{\partial M_{u}}{\partial u}+\frac{\partial N_{\psi}}{\partial v}=H . \Delta_{2} \psi, \quad H d u d v=d \omega
$$


Beltrami: Delle variabili complesse sopra una superficie ecc. 351

quindi :

$$
\Pi=\int\left\{M_{\psi}\left(\frac{d v}{d s}\right)-N_{\psi}\left(\frac{d u}{d s}\right)\right\} \phi d s-\iint \phi \cdot \Delta_{2} \psi \cdot d \omega .
$$

L'integrale semplice può assumerc una forma più comoda. Infatti, pei valori di $M_{\psi}, N_{\psi}$ si ha:

$$
M_{\psi} \frac{d v}{d s}-N_{\psi} \frac{d u}{d s}=\frac{1}{H}\left\{\left(F \frac{d u}{d s}+G \frac{d v}{d s}\right) \frac{\partial \psi}{\partial u}-\left(E \frac{d u}{d s}+F \frac{d v}{d s}\right) \frac{\partial \psi}{\partial v}\right\} .
$$

Ora se s'indicano con $\delta u, \delta v$ gli incrementi che ricevono $u$ e $v$ passando dal punto $(u, v)$ del contorno ad un punto infinitamente vicino nella direzione normale interna all'area, e con $\delta n$ la distanza di questi due punti, si ha (Art. II)

$$
\begin{aligned}
& \frac{E d u+F d v+i H d v}{d s}=-i \frac{E \delta u+F \delta v+i H \delta v}{\delta n}, \\
& \frac{F d u+G d v+i H d v}{d s}=i \frac{F \delta u+G \delta v+i H \delta v}{\delta n},
\end{aligned}
$$

donde :

$$
\begin{array}{ll}
E \frac{d u}{d s}+F \frac{d v}{d s}=H \frac{\delta v}{\delta n}, & F \frac{d u}{d s}+G \frac{d v}{d s}=-H \frac{\delta u}{\delta n}, \\
E \frac{\delta u}{\delta n}+F \frac{\delta v}{\delta n}=-H \frac{d v}{d s}, & F \frac{\delta u}{\delta n}+G \frac{\delta v}{\delta n}=H \frac{d u}{d s} .
\end{array}
$$

Di qui si trae:

$$
M_{\psi} \frac{d v}{i s}-N_{\psi} \frac{d u}{d s}=-\left(\frac{\partial \psi}{\partial u} \frac{\delta u}{\delta n}+\frac{\partial \psi}{\partial v} \frac{\delta v}{\delta n}\right)=-\frac{\delta \psi}{\delta n},
$$

e quindi finalmente:

$$
-\Pi=\int \phi \frac{\delta \psi}{\delta n} \cdot d s+\iint \phi \cdot \Delta_{2} \psi \cdot d \omega
$$

formola in cui l'integrale duplicato è esteso a tutta l'area $\omega$ e l'integrale semplice a tutto il contorno (il quale ora non è piủ vincolato a dover essere persorso in un senso determinato, perchè nella funzione da integrare non figura più l'arco $s$ finito).

Se si fosse considerata la funzione $\psi$ al posto della $\phi$ e reciprocamente, si sarebbe trovato, in modo analogo,

$$
-\Pi=\int \psi \frac{\delta \psi}{\delta n} d s+\iint_{\delta} \psi \cdot \Delta_{2} \phi \cdot d \omega
$$


Beltrami: Delle variabili complesse sopra una superficie ecc.

confrontando i due risultati si ottiene quindi:

$$
\iint\left(\phi . \Delta_{2} \psi-\psi \cdot \Delta_{2} \phi\right) d\left(\nu+\int\left(\phi \frac{\delta \psi}{\delta n}-\psi \frac{\delta \varphi}{\delta n}\right) d s=0,\right.
$$

formola molto importante, la quale esprime, rispetto alle superficie riferite a coordinate curvilinee qualunque, il teorema correlativo di uno notissimo rispetto allo spazio di tre dimensioni riferito a coordinate ordinarie.

Questa formola rimane cosi dimostrata per un'area o soggetta alle restrizioni suenunciate. Ma se si immagina che una formola analoga venga scritta per ciascuna delle aree $\omega$ in cui fu suddivisa l'area $\Omega^{\prime}$ col mezzo delle trasversali $\Lambda^{\prime}$, facendo la somma di tutti i risultati, spariscono gli integrali lineari relativi alle trasversali, poichè ciascun d'essi figura duc volte, con segno contrario in causa dell'opposta direzione della normale $n$. Si ottiene dunque una formola di eguale aspetto, relativa all' intiera area $\Omega^{\prime}$. Anche la formola (22) ̀̀ evidentemente suscettibile di un' eguale estensione.

All' equazione (23) si puó pervenire altresi nel modo seguente.

Consideriamo una delle aree elementari $\omega$, ed una funzione $w$, monodroma finita e continua in tutti i punti di essa. Nelle ipotesi già fatte precedentemente si ha:

$$
\begin{gathered}
\int \frac{\partial w}{\partial u} d u=u_{b}-w_{a} \\
\iint \frac{\partial w}{\partial u} d u d v=\int w_{b} d v_{b}-\int w_{a} d v_{a}=\int w\left(\frac{d v}{d s}\right) d s
\end{gathered}
$$

ed analogamente:

$$
\iint \frac{\partial w}{\partial v} d u d v=-\int w\left(\frac{d u}{d s}\right) d s
$$

formole nolle quali gli integrali duplicati sono estesi a tutta l'area $\omega$, gli integrali semplici al suo contorno, percorso nel senso positivo. In luogo di queste formole si possono, per le (21), scrivere le seguenti :

$$
\begin{aligned}
& \iint \frac{\partial w}{\partial u} d u d v=-\int\left(E \frac{\delta n}{\delta n}+F \frac{\delta v}{\delta n}\right) \frac{w}{H} d s, \\
& \iint \frac{\partial w}{\partial v} d u d v=-\int\left(F \frac{\delta u}{\delta n}+G \frac{\delta v}{\delta n}\right) \frac{w}{H} d s,
\end{aligned}
$$

più simmetriche, benchè meno scmplici, e nelle quali gli integrali lineari 
Beltrami: Delle variabili complesse sopra una superficie ece. 3 su3

sono indipendenti dal modo in cui viene percorso il contorno. Si ponga ora:

$$
\begin{array}{ll}
\text { nella prima formola } & w=\phi M_{\psi}-\psi M_{\varphi}, \\
\text { nella seconda } & w=\phi N_{\psi}-\psi N_{\varphi},
\end{array}
$$

valori i quali suppongono monodrome finite e continue non solo lc funzioni $\phi$ e $\psi$, ma anche le loro derivate prime, che entrano nelle $M, N$. Sommando i risultati ed osservando che si ha:

$$
\begin{gathered}
\frac{\partial\left(0 M_{\psi}-\psi M_{\varphi}\right)}{\partial u}+\frac{\partial\left(\varphi N_{\psi}-\psi N_{\varphi}\right)}{\partial v}=\left(\frac{\partial M_{\psi}}{\partial u}+\frac{\partial N_{\psi}}{\partial v}\right) \phi-\left(\frac{\partial M_{\varphi}}{\partial u}+\frac{\partial N_{\varphi}}{\partial v}\right) \psi \\
=H\left(\phi . \Delta_{2} \psi-\psi \cdot \Delta_{2} \phi\right),
\end{gathered}
$$

ed inoltre che :

$$
E M_{\varphi}+F N_{\Psi}=H \frac{\partial \varphi}{\partial u}, \quad F M_{y}+G N_{\varphi}=H \frac{\partial \psi}{\partial v}
$$

si ritrova immediatamente l'equazione (23).

Questo processo non conduce, come il già usato, alla formola (22) che è interessante a conoscersi. 亡̀ perd facile ricavare questa formola dalla (23). Infatti scriviamo dapprima quest' ultima come segue:

$$
\iint p \cdot \Delta_{2} \psi \cdot d \omega+\phi \int \frac{\delta^{\prime}}{\delta n} d s=\iint \psi \cdot \Delta_{2} p \cdot d \omega+\psi \int \frac{\delta_{p}}{\delta n} d s .
$$

Poi ossertiamo che dalla stessa (23), per $\phi=1, \psi=2 v$, si deduce:

$$
\iint_{\imath} \Delta_{2} 2 v \cdot d w+\int \frac{\delta w}{\delta n} d s=0
$$

F lecito porre in questa formola $w=p . \psi$, onde s'ottiene:

ossia :

$$
\iint\left\{\phi \cdot \Delta_{2} \psi+\psi \cdot \Delta_{2} \phi+2 \Delta_{1} \eta \psi\right\} d \omega+\int\left\{\phi \frac{\delta \psi}{\delta n}+\psi \frac{\delta p}{\delta n}\right\} d s=0
$$

$-\iint \Delta_{1} \phi \psi \cdot d \omega=\frac{1}{2}\left\{\iint \phi \cdot \Delta_{2} \psi \cdot d \omega+\int \phi \frac{\delta \psi}{\delta n} d s\right\}+\frac{1}{2}\left\{\iint \psi \cdot \Delta_{2} \phi \cdot d \omega+\int \psi \frac{\delta \varphi}{\delta n} d s\right\}$.

$\mathrm{Ma}$ in virtù della (24) le due espressioni componenti il secondo membro sono cguali fra loro; quindi si puó, in luogo dolla loro somma, scrivere il doppio dell' una o dell'altra. In tal modo si ricade appunto sulla formoli (22) o sulla $\left(22^{\prime}\right)$. 
Quando $\phi=\psi$ dalla (22) si ha:

$$
-\iint \Delta_{1} \phi \cdot d()=\iint \phi \cdot \Delta_{2} \phi \cdot d \omega+\int \phi \frac{\delta \varphi}{\delta n} d s
$$

equazione nella quale è importante il notare che gli elementi $\Delta_{1} \phi$.do del primo integrale duplicato sono tutti essenzialmente positivi, in forza del significato che ha il primo parametro differenziale della funzione $\phi$ (Art. I). Su questa proprietà si appoggia la dimostrazione delle note proprietà delle funzioni $\phi$ che rendono $\Delta_{2} \phi=0$, mediante l' uso del principio di Dirichlet, come si suol fare per il piano.

VI.

Supponiamo che la funzione $\psi$ dell' art. prec. diventi, in un certo punto $O$, infinita come $\log \frac{1}{r^{*}}, r$ essendo la distanza geodetica di questo punto da un punto qualunque della superficie; c propriamente ammettiamo che, per distanze geodetiche sufficientemente piccole, si abbia:

$$
\psi=\log \frac{1}{r}+r^{\nu} Q
$$

dove $\nu$ è un esponente maggiore di zero e $Q$ una quantità che, per $r=0$, non diventa nè nulla nè infinita (a meno che non sia sempre=0), e che in gencrale dipende dalle coordinate del punto a cui corrisponde il valore di $\psi$ che si considera.

Se il punto $O$ ì interno all' area $\Omega^{\prime}$, il teorema (23) non puó più essere applicato a quest'area; ma diventa applicabile all'area che si ottiene to-glicndo ad $\Omega^{\prime}$ una porzione, piccola quanto si vuole, circostante al punto $O$. Riterremo che questa porzione sia limitata da una circonferenza geodetica di raggio $r$ piccolissimo, col centro nel punto $O$. In tale ipotesi la formola (23) continuerà a sussistere, purchè si aggiungano al suo primo mombro i termini seguenti:

$$
-\iint\left(\phi \cdot \Delta_{2} \psi-\psi^{\prime} \cdot \Delta_{2} \phi\right) d \omega^{\prime}+\int\left(\phi \frac{\partial^{\prime}}{\partial n^{\prime}}-\frac{\partial^{\prime}}{\partial \nu^{\prime}}\right) d s^{\prime}
$$

dove $d \omega^{\prime}, d n^{\prime}, d s^{\prime}$ fanno l'ufício di $d(\omega, d n, d s$ relativamente all' aroa ed al contorno del piccolo cerchio geodetico. 
Beltrami: Delle variabili complesse sopra una superficie ece. 35̈

Per calcolare il valore della precedente espressione conviene ricorrero ad una forma dell' clemento lineare appropriata al caso attuale, cioc̀ alla forma

$$
d s^{2}=d r^{2}+R^{2} d \varepsilon^{2}
$$

che risulta dall' assumero come curve coordinate le linee geodetiche divergenti dal punto $O$ e le circonferenze geodetiche che hanno il centro nel medesimo punto: $r$ è la lunghezza di un arco di goodetica contato da $O$, $\varepsilon$ è l'angolo che una delle geodetiche, presa come orjogine, fia con una qualunque delle altre. In queste condizioni $\dot{e}$ facile vedere che $R$ i goneralmonte della forma:

$$
R=r\left(1+r^{\mu} P\right)
$$

dove $\mu$ o un esponente maggioro di zero e $P$ una funziono di $r$ o di $\varepsilon$ che per $r=0$ non diventa nè nulla nè infinita $\left(^{*}\right)$. Si lıa poi:

$$
d \omega^{\prime}=R d r d \varepsilon, \quad d n^{\prime}=d r, \quad d s^{\prime}=R d \varepsilon,
$$

epperò l'espressione da calcolarsi, ritenuta continua la funzione $\phi$ e le sue derivato, diventa:

$$
-\bar{\phi} \iint R \cdot \Delta_{2} \psi \cdot d r d \varepsilon+\overline{\Delta_{2} \phi} \iint R \psi d r d \varepsilon+\bar{\phi} \int R \frac{\partial \psi}{\partial r} d \varepsilon-\overline{\frac{\partial \varphi}{\partial r}} \int R \psi d \varepsilon
$$

dove $\overline{c^{\prime}}, \overline{\Delta_{2} \phi}, \ldots$ sono certi valori medii delle funzioni $\phi, \Delta_{2} \phi, \ldots$ convenientemente scelti fra quelli che queste funzioni prendono entro i limiti degli integrali donde sono levate fuori.

Ora ossorviamo che:

$\left.1^{0}\right)$ Avendosi, por la forma (27) dell' elemento lineare,

$$
\Delta_{2} \psi=\frac{1}{R}\left\{\frac{\partial}{\partial r}\left(R \frac{\partial \psi}{\partial r}\right)+\frac{\partial}{\partial \varepsilon}\left(\frac{1}{R} \frac{\partial \psi}{\partial \varepsilon}\right)\right\},
$$

c $\downarrow$ avendo la forma (20), se si pono per brovità :

$$
\left(1+r^{\mu} \mu^{\mu}\right)\left(-1+r^{\mu} Q+r^{\nu+1} \frac{\partial Q}{\partial r}\right)+1=K, \frac{1}{1+r^{\mu} P} \frac{\partial Q}{\partial \varepsilon}=K_{1},
$$

si trova:

$$
\Delta_{2} \psi=\frac{1}{R}\left\{\frac{\partial K}{\partial r}+r^{\nu-1} \frac{\partial K_{1}}{\partial \varepsilon}\right\}
$$

() Gauss, Disquisitiones generales circa superficies curvas, Art. XIX.-Il aaso di $P=0$, cioè di $K=r$, non si vorilica che per lo superficie applicabili sopra un piano. 
Di qui, moltiplicando per $R d r d \varepsilon$ ed integrando fra i limiti 0 ed $r, 0$ ed $\varepsilon$, si deduce:

$$
\iint R \cdot \Delta_{2} \psi \cdot d \omega^{\prime}=\int_{0}^{\varepsilon} K d \varepsilon+\frac{1}{\nu} \int_{0}^{r} K_{1} d\left(r^{\nu}\right)-\frac{1}{\nu} \int_{0}^{r} K_{1}^{0} d\left(r^{\nu}\right)
$$

dove $K_{1}^{0}$ è il valore di $K_{1}$ per $\varepsilon=0$. Ora, in virtù del valore di $K$, è evidente che l'integrale relativo ad $\varepsilon$ può essere decomposto in più integrali simili, ciascuno dei quali è moltiplicato per una potenza positiva di r. - Quanto ai due integrali relativi ad $r^{\nu}$, e chiaro che chiamando $c$ e $c^{0} \mathrm{i}$ valori numericamente più grandi che lo quantità $K_{1}, K_{1}^{0}$ assumono fra i limiti 0 ed $r$, gli integrali stessi rimangono numericamente minori delle due quantiti $\frac{c r^{\nu}}{\gamma}$ e $\frac{c^{0} r^{\nu}}{\gamma}$ rispettivamente. - Di qui si conclude che l'integrale:

$$
\iint R \cdot \Delta_{2} \psi \cdot d \omega^{\prime}
$$

converge verso zero per $r=0$, e cio qualunque sia il limito, superiorc $\varepsilon$ della integrazione relativa ad $\varepsilon$. Lo stesso ha luogro quando $Q$ è sempro $=0$, ciò̀ quando $\psi=\log \frac{1}{r}$.

$\left.2^{\circ}\right)$ La quantità:

$$
R \psi=\left(1+r^{\mu} P\right) \cdot r \log \frac{1}{r^{2}}+r^{\nu+1}\left(1+r^{\mu} P\right) Q,
$$

converge a zoro quando $r$ diventa evanescente; quindi, per tutti i valori di $r$ inferiori ad un certo limite, essa si mantiene numericamente minoro di una quantita $\rho$, la quale si annulla con $r$. Dunque il valor numerico degli integrali :

$$
\iint R \nsucc d r d \varepsilon, \quad \quad \int R \psi d r
$$

estesi fra 0 od $r, 0$ ed $\varepsilon$, ̀̀ rispettivamente minore di :

$$
\rho r \varepsilon, \quad \rho \varepsilon,
$$

epperd ambeduc gli integrali si annullano con $r$.

$3^{\circ}$ ) Rammentando l'espressione poc'anzi indicata con $K$, si ha:

$$
R \frac{\partial \psi}{\partial r}=K-1
$$


Beltrami: Delle variabili complesse sopra unu superficie ecc. 357

c quindi;

$$
\int_{0}^{\varepsilon} R \frac{\partial \psi}{\partial r} d \varepsilon=\int_{0}^{\varepsilon} K d \varepsilon-\varepsilon
$$

poichè dunque si è veduto già che $\int_{0}^{\varepsilon} K d \varepsilon$ converge verso zero per $r=0$, si riconosce immediatamente che, per lo stesso valore di $r$, si ha:

$$
\int_{0}^{\varepsilon} R \frac{\partial \psi}{\partial r} d \varepsilon=-\varepsilon
$$

Se si raccolgono le proprietà qui notate dei varii integrali che compongono l'espressione di cui si cercava il valore, nella quale le integrazioni relative ad $\varepsilon$ andavano estese fra $0 \mathrm{e} 2 \pi$, si vede subito che, per $r$ evanesccnte, quell'espressione converge verso il valore $-2 \pi p_{0}$, $\phi_{0}$ essendo il valore che riceve la funzione $\phi$ nel punto $O$. In conseguenza si ha la formola:

$$
2 \pi p_{0}=\iint\left(\phi \cdot \Delta_{2} \psi-\psi \cdot \Delta_{2} \phi\right) d \omega+\int\left(\phi \frac{\delta \psi}{\delta n}-\psi \frac{\delta \phi}{\delta n}\right) d s
$$

purchè la funzione $\psi$ abbia il carattere suindicato in un punto $O$ dell'area $\Omega^{\prime}$ alla quale si estendono le integrazioni, e quindi in particolare quando $\psi=\log \frac{1}{r^{2}}$.

Se il contorno dell' area $\Omega^{\prime}$ passasse pel punto $O$, singolare per la funzione $\psi$, converrebbe scansarlo descrivendo intorno ad esso come centro un arco

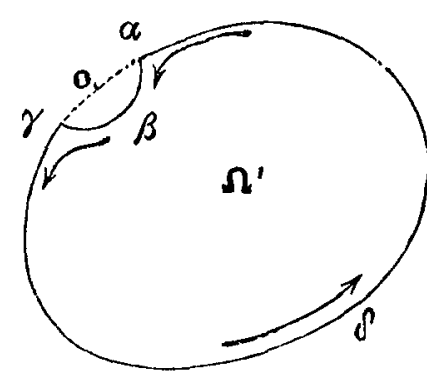
di cerchio geodetico, di raggio piccolissimo, $\alpha \beta \gamma$, e sostituendo questo arco alla porzione $\alpha \gamma$ di contorno che comprende il punto $O$. L'arco anzidetto avrà l'ampiezza di $180^{\circ}$ quando $O$ sarà un punto ordinario del contorno, ed avrà un'ampiezza $\varepsilon$ differente da $180^{\circ}$ se $O$ sarà un punto angoloso del contorno stesso. In quest' ultimo caso si trova immediatamente, colla scorta delle precedenti osservazioni, che al posto della (28) si ha la formula:

$$
\varepsilon \phi_{0}=\iint\left(\phi . \Delta_{2} \psi-\psi \cdot \Delta_{2} \phi\right) d \omega+\int\left(\phi \frac{\delta \psi}{\delta n}-\psi \frac{\delta \varphi}{\delta n}\right) d s,
$$

dove l'integrale duplicato si riferisce all'area contenuta entro il contorno $\alpha \beta \gamma \delta \alpha$, e l'integrale semplice al contorno medesimo, ommesso l'elemento 
nel quale si trova il punto $O$. Si troverebbe un cguale risultato descrivendo un piccolo arco esteriormente all'area, e prendendo quindi lo mosse dall' 0 quazione (28) anzichè dalla (23).

Per $\phi=1$ le (28), (29) danno:

$$
\iint \Delta_{2} \psi \cdot d(r)+\int \frac{\delta \psi}{\delta n} d s=2 \pi, \quad \text { oppure }=\varepsilon
$$

secondo che il punto $O$, dove la funzione $\psi$ diventa infinita, e interno all'arca $\Omega^{\prime}$, ovvero situato in un punto del contorno in cui duc elementi contigui fanno l'angolo $\varepsilon$. È facile vedere che questo ultimo caso è il generale, ed abbraccia quello del punto interno e del punto esterno. Infatti, in queste due ultime ipotesi, si può aggiungere al primitivo contorno una linea che vada da un punto $A$ del contorno stesso al punto $O$ e che ritorni poscia sopra sè stessa a raggiungere nel punto $A$ il contorno: con cio non si alterano

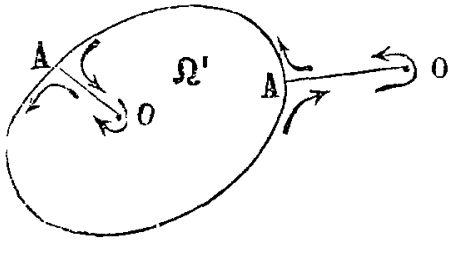
nè gli integrali d'area, nè quelli di contorno. Ma considerando questa linea doppia come facente parte del contorno, è chiaro che l'angolo, interno all'area, dei due elementi sovrapposti che terminano in $0, \grave{\mathrm{e}}=0$ quando $O \dot{\mathrm{c}}$ esterno all'area, ed $=2 \pi$ quando $O$ è interno. - Ciò vale anche per la (29).

F utile fare la seguento osservazione circa il valore dell' esponente positivo $\mu$, che figura nel valore di $R$. Il prodotto reciproco dei duc raggi di curvatura principali è dato, come si sa, dalla formola:

$$
-\frac{1}{R} \frac{\partial^{2} R}{\partial r^{2}}=-\frac{r^{\mu-2}}{1+r^{\mu} P}\left\{\mu(\mu+1) P+2(\mu+1) r \frac{\partial P}{\partial r}+r^{2} \frac{\partial^{2} P}{\partial r^{2}}\right\}
$$

quindi il suo valore per $r=0$, cioè nel punto $O$, ̀̀ nullo, finito od infinito secondo che $\mu$ ̀े $>2,=2$, oppure $<2$. Quando dunque l'esponente $\mu$, positivo, ¿ minore di 2 , la superficie non ha nel punto $O$ una curvatura ordinaria.

la formola (28) esprime, rispetto alle superficie, un teorema analogo a quello di Green per lo spazio di tre dimensioni. 
Beltrami: Delle variabili complesse sopra una superficie ecc. 350

VII.

Poichè il binomio $x(U d u+V d v)$ dev' essere un differenziale esatto, si deve avere:

$$
\frac{\partial(* U)}{\partial v}=\frac{\partial(x V)}{\partial u}
$$

ossia :

$$
U \frac{\partial \log x}{\partial v}-V \frac{\partial \log x}{\partial u}==\frac{\partial V}{\partial u}-\frac{\partial U}{\partial v}
$$

Ponendo in questa equazione, ed in quella che se ne ottiene moltiplicandola per $V^{\prime}$, i valori (4) di $U, V, V^{\prime}$, si trovano le due formole equivalenti :

$$
\begin{aligned}
& E \frac{\partial \log x}{\partial v}-(F+i H) \frac{\partial \log x}{\partial u}=W U, \\
& G \frac{\partial \log x}{\partial u}-(F-i H) \frac{\partial \log x}{\partial v}=-W V^{\prime},
\end{aligned}
$$

dove per brevità si è posto:

$$
W=\frac{\partial V}{\partial u}-\frac{\partial U}{\partial v}
$$

Le precedenti due equazioni si possono scrivere come segue.

$$
\begin{aligned}
& \frac{G \frac{\partial \log x}{\partial u}-F \frac{\partial \log x}{\partial v}}{H}=-i \frac{\partial \log x}{\partial v}-\frac{W V^{\prime}}{I I}, \\
& \frac{E \frac{\partial \log x}{\partial v}-F \frac{\partial \log x}{\partial u}}{H}=i \frac{\partial \log x}{\partial u}+\frac{W U}{I I},
\end{aligned}
$$

donde si deducc, rammentando l'espressione della funzione $\Delta_{2} \log x$ (Art. I),

$$
\Delta_{2} \log x=\frac{1}{I I}\left\{\frac{\partial}{\partial v}\left(\frac{W U}{H}\right)-\frac{\partial}{\partial u}\left(\frac{W V^{\prime}}{I I}\right)\right\}
$$

equaziono notevolissima, in quanto manifesta che il valore della funzione $\Delta_{2} \log x$ può esscre ottenuto mediante le sole quantita $E, F, G$, che carat1crizzano l'elemento linearo della superficie, insieme collo loro derivate. 
Chiamando $k$ il modulo della funzione $x$, si deduce dalla precedente equazione, calcolando la parte reale del secondo membro,

$$
\Delta_{2} \log k=-\frac{1}{2 H}\left\{\frac{\partial}{\partial u}\left[\frac{1}{H}\left(\frac{\partial G}{\partial u}-\frac{F}{E} \frac{\partial E}{\partial v}\right)\right]+\frac{\partial}{\partial v}\left[\frac{1}{H}\left(\frac{\partial E}{\partial v}-2 \frac{\partial F}{\partial u}+\frac{F}{E} \frac{\partial E}{\partial u}\right)\right]\right\},
$$

formola nella quale si riconosce l'espressione data da Lrouvich per la misura della curvatura. Abbiamo già mostrata altrove la ragione d'essere di questo risultato (*).

Consideriamo un' area $\Omega^{\prime}$ nella quale $k$ si mantenga finita e maggiore di zero: applicando ad essa la formola $\left(24^{\prime}\right)$, dopo avervi posto $w=\log k$, si trova:

$$
\iint d \omega \cdot \Delta_{2} \log k=-\int d s \frac{\delta \log k}{\delta n} \text {. }
$$

Il primo membro non è che la curvatura integra o totale della porzione $\Omega^{\prime}$ di superficie: denominandola $\Gamma^{\prime}$, si ha quindi:

$$
\Gamma^{\prime}=-\int d s \frac{\delta \log k}{\delta n}
$$

formola nella quale l'integrale del secondo membro è esteso a tutto il contorno dell' area considerata.

Bisogna osservare che, mercè questa formola, le aree sferiche $\Gamma^{\prime}$, che secondo la teoria di GAUss servono a misurare la curvatura totale, ricevono un segno determinato. Infatti l'elemento dell'integrale doppio $\Gamma^{\prime} \grave{\mathrm{c}} \frac{d \omega}{R_{1} \Pi_{2}}, R_{1}$ ed $R_{2}$ essendo i due raggi principali di curvatura della superficie, e quindi l'elemento stesso è positivo o negativo secondo che i due raggi anzidetti hanno la stessa direzione o direzioni contrarie: quindi l'area sferica $\Gamma^{\prime}$ risulta generalmente formata dalla somma algebrica di più parti aventi segni diversi. Ne consegue che più porzioni contigue di superficie possono avere, separatamente considerate, una curvatura finita, mentre il loro complesso potrà avere una curvatura totale nulla.

La quantità $\frac{\delta \log k}{\delta n}$ è suscettibile di una trasformazione notevole. Chiamanùo $\frac{1}{p}$ la curvatura geodetica di una linea qualunque $s$ tracciata sulla superficie

() Ricerche d'analisi applicata alla geometria, nel Giornale matematien di Napoli. 
Beltrami: Delle rariabili complesse sopra una superficie ece. $\quad 361$

il cui elemento lineare è della forma:

$$
\frac{d p^{2}+d q^{2}}{k^{2}}
$$

(forma che conviene appunto, pel significato di $k$, alla superficie da noi considerata), si ha da formole note $\left(^{*}\right)$ :

$\operatorname{Ma}(21)$

$$
\frac{1}{\rho}=\frac{1}{l i^{2}}\left(\frac{d p}{d s} \frac{d^{2} q}{d s^{2}}-\frac{d q}{d s} \frac{d^{2} p}{d s^{2}}\right)+\frac{\partial \log k}{\partial q} \frac{d p}{d s}-\frac{\partial \log l}{\partial p} \frac{d q}{d s}
$$

$$
\frac{d p}{d s}=\frac{\delta q}{\delta n}, \quad \frac{d q}{d s}=-\frac{\delta p}{\delta n}
$$

quindi :

$$
\frac{1}{p}=-\frac{1}{l^{2}}\left(\frac{d p}{d s} \frac{d^{2} q}{d s^{2}}-\frac{d q}{d s} \frac{d^{2} p}{d s^{2}}\right)+\frac{\delta \log l}{d n}
$$

Chiamiamo $\eta$ l'angolo che l'arco $s$ fa colla curva $u$, od avremo evidentemente:

donde :

$$
\frac{d p}{d s}=k \cos n, \quad \frac{d q}{d s}=k \operatorname{sen} \eta
$$

dunque:

$$
\frac{d p}{d s} \frac{d^{2} \varphi}{d s^{2}}-\frac{d q}{d s} \frac{d^{2} p}{d s^{2}}=l l^{2} \frac{d n}{d s^{2}}
$$

$$
\frac{1}{p}=\frac{d n}{d s}+\frac{\delta \log l}{\delta n}
$$

Questa formola dà a $\rho$ il segno positivo od il negativo, secondo che $\rho \grave{e}$ diretto nel senso di $\delta n$ od in senso contrario.

Chiamando di l'angolo di contingenza geodetica, cioè ponendo:

$$
d \tau=\frac{d s}{i}
$$

si deduce dalla precedente questa relazione:

$$
-\frac{\delta \log k}{\delta n}=d \eta-d \tau
$$

dunque, sostituendo nella (34),

$$
\Gamma^{\prime}=E-T
$$

() Citate Ricerche. 
Beltrami: Delle variabili complesse sopra una superficie ecr.

dove $E$ e $T$ sono le somme, relative a tutto il contorno $s$, degli angoli $d n$ e $d \tau$ corrispondenti a questo contorno stesso.

Per determinare con sicurezza queste somme in tutti i casi, conviene far in modo che in nessun punto del contorno gli angoli $d n, d \tau$ sieno finiti; cioè conviene togliere i punti angolosi del contorno, se ve ne sono, sostituendovi delle piccole curve d'accordo, che tolgano le discontinuità dell'angolo $\eta$ e impediscano al raggio $\rho$ di essere nullo in qualche punto. Quanto all' angolo $\tau$ è bene osservare che, in virtù della convenzione circa il segno di $\rho$, i suoi elementi infinitesimi $d \tau$ sono positivi o negativi, secondo che le due geodetiche, tangenti consecutive al contorno, dalle quali sono formati, si incontrano esternamente od internamente all' area $\Omega^{\prime}$. Supporremo anche, in quel che segue, che quest'area sia semplicemente connessa.

La somma $\int d \eta=E$ si eseguisce agevolmente. Infatti dividiamo dapprima l'area $\Omega^{\prime}$, come nell' art. $V$, in un certo numero di pezzi $\omega$, ciascuno dei quali abbia il proprio contorno attraversato in due soli punti dalle curve $u$. Sieno $v=v_{0}, v=v_{1}$, le due curve $u$ entro le quali è racchiuso uno di questi pezzi (vedi la figura dell'art. V). Nel punto di contatto $\alpha$ colla prima curva l'angolo $\eta$ è nullo, e da questo punto, variando uniformemente, va ad acquistare il valore $\pi$ nel punto di contatto $\gamma$ colla seconda; poscia cresce da $\pi$ a $2 \pi$, quando si ritorna per $\delta$ al primitivo punto $\alpha$. Dunque per questo pezzo la somma in discorso è $2 \pi$, epperò, chiamando $T_{\omega}$ la somma algebrica de gli angoli di contingenza geodetica, si ha per esso:

$$
\Gamma_{\omega}^{\prime}=2 \pi-T_{(1)} \text {. }
$$

Per un secondo pezzo si avrà analogamente:

$$
\Gamma^{\prime} \omega_{1}=2 \pi-T_{\omega_{1}}
$$

e così di seguito. Ora torniamo a ricongiungere i varii pezzi $\omega, \omega_{1}$, ecc.,

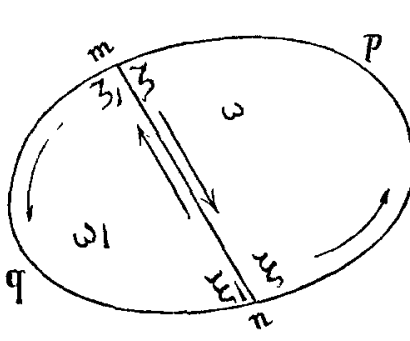
per il che basterà considerare il risultato della congiunzione di due pezzi $\omega, \omega_{1}$ aventi in comune una parte $m n$ del loro contorno. Chiamiamo $T_{(w)}^{\prime} T^{\prime}{ }_{()_{1}}$ ls parti di $T_{\omega}, T_{\omega_{1}}$ relative alle curve npm, mqn rispettivamente, e $t$ quella relativa alla trasversale mn, parte che è comune ai due contorni, ma con segno contrario. Si avrà:

$$
T_{w}=T^{\prime}+t+\check{\zeta}_{1}+\zeta_{1}, \quad T_{()_{1}}=T_{m_{1}}^{\prime}-t+\xi+\zeta,
$$


Beltrami: Delle variabili complesse sopra una superficie ecc. $\mathbf{3 6 3}$

quindi :

$$
T_{\omega}+T_{\omega_{1}}=T^{\prime \prime}+T^{\prime \prime} \omega_{1}+2 \pi=T_{\omega_{+} \omega_{1}}+2 \pi .
$$

Ne risulta che, sommando le due formole precedentemente ottenute, si ha:

$$
\Gamma_{\left(\omega+\omega_{1}\right.}^{\prime}=2 \pi-T_{(1)+\omega_{1}}
$$

formola analoga alle anzidette, ma relativa al complesso delle due parti $\omega$ ed $\omega_{1}$. Così continuando si trova finalmente:

$$
\Gamma^{\prime}=2 \pi-T
$$

dove $T$ è la somma di tutti gli angoli di contingenza geodetica relativi al contorno dell'area $\Omega^{\prime}$. (Donde si conclude eziandio che, nelle condizioni ammesse circa alla disposizione delle curve coordinate, si ha in ogni caso, per un contorno chiuso, $E=2 \pi$.)

La proprietà espressa dalla equazione precedente è stata indicata, sotto forma un po' diversa, dal sig. Boxnet. (*)

Se il contorno è un poligono geodetico, sono nulle, nella somma $T$, tutte le parti relative ai lati successivi di esso, e non rimangono che quelle dovute alle piccole curve d'accordo che abbiam detto doversi sostituire agli angoli del poligono, cioè non rimane che la somma delle deviazioni di ciascun lato sul precedente. Chiamando dunque $\Delta$ queste deviazioni, si ha:

$$
\Gamma^{\prime}=2 \pi-\Sigma \Delta
$$

formola che esprime un celebre teorema di Gauss $(* *)$.

Se la superficie è riferita ad un sistema di coordinate geodetiche $r$ ed $\varepsilon$, come si suppose nell' art. precedente, in prossimita del punto $O$ dal quale divergono le geodetiche l'espressione dell'elemento lineare si può scambiare colla seguente:

$$
d s^{2}=d r^{2}+r^{2} d \varepsilon^{2}
$$

per la quale il modulo $k$ del fattore d'integrazione $*$ può porsi $=\frac{1}{r}$. Ne risulta che la funzione logk diventa, in questo caso, infinita in $O$ come

(*) Journul de l'Ecole Polytechnique, cahier 41.

(*) Disquisitiones generales etc. art. XX. 
$\log \frac{1}{r}$. Se dunque si suppone che il punto $O$ sia nell'interno dell' area $\Omega^{\prime}$, bisogna sostituire la formola:

$$
\Gamma^{\prime}=\iint_{e} d \omega \Delta_{2} \log k=2 \pi-\int d s \frac{\delta \log k}{\delta n}
$$

[dedotta dalla (30)] a quella da cui siamo partiti per istabilire la (37). Si trova così :

$$
\int d s \frac{\delta \log k}{\delta n}=T
$$

mentre, nelle ipotesi ammesse precedentemente, si aveva:

$$
\int d s \frac{\delta \log k}{\delta n}=T-2 \pi
$$

Di qui si conclude l'interessante proprietà che: l'integrale $\int d s \frac{\delta \log k}{\delta n}$, esteso ad un contorno chiuso, è eguale a $T-2 \pi$, oppure a $T$, secondo che il punto in cui la funzione $k$ diventa infinita come $\frac{1}{r^{2}}$, è interno od esterno all' area chiusa da quel contorno.

Nel piano, riferito a coordinate polari ordinarie, si ha $k=\frac{1}{r}, T=2 \pi$, quindi la proprietà precedente fornisce, come caso particolare, il teorema, facilissimo a verificarsi, che : se $r$ è la clistanza di un punto fisso del piano da un punto variabile lungo un contorno chiuso, l'integrale $\int \frac{\delta \log r}{\delta n} d s$ esteso a questo contorno, $\grave{e}=0$ oppure $=2 \pi$, secondo che il punto fisso è esterno od interno all' area limitata dal contorno.

Un caso particolare interessante della formola (3\%) si ottiene supponendo che la curva $s$ sia una di quelle dotate della proprietà di contenere, sotto un dato perimetro, la massima area, o di avere, per una data area racchiusa, il minimo perimetro. E noto infatti che per queste curve la curvatura geodetica $\frac{1}{\rho}$ ¿ dovunque costante, e che quindi $T=\frac{s}{\rho}, s$ essendo il perimetro totale: dunque si ha, per la curvatura totale dell' area racchiusa,

$$
\Gamma^{\prime}=2 \pi-\frac{s}{p} .
$$


Beltrami: Delle variabili complesse sopra una superficie ecc. 36 ä

Il valore della curvatura totale può anche essere espresso facilmente per un integrale lineare, dipendente dalle sole $E, F, G$ e loro derirate. Si ponga infatti, per brevità,

$$
\frac{1}{2 H}\left(\frac{F}{E} \frac{\partial E}{\partial v}-\frac{\partial G}{\partial u}\right)=Q, \quad \frac{1}{2 H}\left(\frac{\partial E}{\partial v}-2 \frac{\partial F}{\partial u}+\frac{F}{E} \frac{\partial E}{\partial u}\right)=P,
$$

e la (33) diverrà :

$$
\Delta_{2} \log k=\frac{1}{H}\left\{\frac{\partial Q}{\partial u}-\frac{\partial P}{\partial v}\right\} .
$$

Quindi, moltiplicando $\mathrm{per}^{\circ} d_{\omega}=H d u d v$, ed integrando al modo che si è fatto verso la fine dell' Art. V, si troverà :

$$
\Gamma^{\prime}=\int(P(u+Q d v)
$$

dove l'integrale del secondo membro è esteso alla curva chiusa che limita la porzione di superficie di cui $\Gamma^{\prime}$ e la curvatura totale.

Si connette con questa espressione di $\Gamma^{\prime}$ una trasformazione notevole che può essere effettuata sulla quantità $\frac{\delta \log x}{\delta n}$. Si ha infatti.

$$
\frac{\delta \log x}{\delta n}=\frac{\partial \log x}{\partial u} \frac{\delta u}{\delta n}+\frac{\partial \log x}{\partial v} \frac{\delta v}{\delta n}
$$

ossia, per le (21),

$$
H \frac{\delta \log x}{\delta n}=\left(E \frac{d u}{d s}+F \frac{d v}{d s}\right) \frac{\partial \log x}{\partial v}-\left(F \frac{d u}{d s}+G \frac{d v}{d s}\right) \frac{\partial \log x}{\partial u} .
$$

Ma essendo, per le (4),

$$
E=U^{2}, \quad F=\frac{U\left(V+V^{\prime}\right)}{2}, \quad G=V V^{\prime}, \quad \frac{i H}{U}=\frac{V-V^{\prime}}{2},
$$

a questa equazione si puó dare la forma seguente:

$$
H \frac{\delta \log x}{\delta n}=\left(U \frac{\partial \log x}{\partial v}-V \frac{\partial \log x}{\partial u}\right)\left(U \frac{d u}{d s}+V^{\prime} \frac{d v}{d s}\right)+i H \frac{d \log x}{d s},
$$

quindi (31):

$$
\frac{\delta \log x}{\delta n} d s=\frac{W\left(U d u+V^{\prime} d v\right)}{H}+i \cdot d \log x
$$


Beltrami: Delle variabili complesse sopra una superficie ecc.

formola che contiene la trasformazione alla quale alludevamo. (La caratteristica $d$ serve sempre a designare spostamenti nel senso dell' arco $s$, mentre la $\delta$ indica spostamenti normali).

Da essa si deduce, integrando lungo una curva chiusa qualunque,

$$
\int \frac{\delta \log \varkappa}{\delta n} d s=\int \frac{W\left(U d u+V^{\prime} d v\right)}{H}+i \int d \log \varkappa
$$

Ma se questa curva è il contorno già considerato nella formola (34), nell'interno del quale abbiamo supposto che $k=\bmod *$ non diventasse mai $=0$, è chiaro che neppure $x$ diventerà zero in esso, epperò si avrà $\int d \log x=0$, e quindi:

$$
\int \frac{\delta \log \alpha}{\delta n} d s=\int \frac{W\left(U d u+V^{\prime} d v\right)}{H}
$$

Eguagliando le parti reali dei due membri di questa equazione, si ricade, per la (34), sulla (39).

Bologna, dicembrc 1867. 